\title{
Time, space, spacetime
}

\section{Barry Dainton: Time and space. Second Edition. Durham: Acumen Publishing Limited, 2010, xvi+464pp, $£ 57$ HC, £17.59 PB}

\section{Dennis Dieks}

(C) The Author(s) 2012. This article is published with open access at Springerlink.com

Anyone who has ever made an attempt to get acquainted with the philosophy of time, starting with McTaggart's classic "The Unreality of Time", will know how easy it is to become bewildered by the subject. Typically, papers in this area are full of arguments that one by one strike the reader as logically compelling or at least plausible ("an event cannot be both future and past"), whereas nevertheless the conclusions one is led to ("time is unreal"; "according to relativity there is no real change"; etc.) strike one as moot at best. It is inevitable to feel a desire for a sober and lucid overview that puts all the relevant themes and premises in order, analyses their precise content, and identifies their strengths and weaknesses. This urge for clarity and impartial assessment can be satisfied since 2001, when Barry Dainton's "Time and Space" first appeared. A second, revised edition of this important book has now come out.

Dainton's book systematically explores the metaphysics and physics of time and space, starting with McTaggart and ending with up-to-the-minute developments in string and membrane theory. The volume can be used as a textbook, but it is more than that: It contains independent analysis on many points and, I would say, is in several cases more authoritative than the primary literature it reviews. For example, whoever wants to know about McTaggart's argument and its validity is advised to look here, rather than to study McTaggart's paper itself or the countless articles that have been written in response to it.

The main theme of the book is the existence of time and space either as "substances", subsisting by themselves (substantivalism), or as representational devices introduced by us to handle relations between material things (relationism). In the case of time, there is the additional important issue of whether time is

D. Dieks $(\bowtie)$

History and Foundations of Science, Utrecht University, POB 80010, 3508 TA Utrecht, The Netherlands e-mail: d.dieks@uu.nl 
dynamic (whether time "flows") or rather is like space, to be represented together with the spatial dimensions in a "static" block universe.

It is one of the highlights of the book that it not only clearly and extensively discusses the various possible positions about the nature of time, with their pros, cons and sometimes inconsistencies, but also offers an analysis of our experience of time flow. This is a neglected subject: Many authors take it as self-evident that a dynamic A-theory of time is needed to do justice to our direct temporal experience, and that A-theory notions immediately explain this experience. However, on second thoughts it is not so clear how such an A-explanation should work. If we put aside the important and notorious difficulty of making sense at all of an objective motion of the Present (motion is change in time; with respect to what should we conceive of a shift of the Now?), we are still facing the question of whether we can be assumed to possess some special organ to register this mysterious motion, if it exists. It is good to see that Dainton devotes a chapter, Time and Consciousness, to questions relating to this phenomenology of time and in particular to the possibility of explaining our time experience within a "static" B-theory of time. Contrary to what may appear at first sight, this explanatory task offers interesting prospects.

The most substantive additions to the original text, in this new edition, are two new chapters on Zeno's paradoxes, and two sections on "dark matter" and "dark energy", respectively. The latter topics certainly help to keep the book up-to-date: They reflect recent developments in our thinking about what General Relativity tells us about the spatiotemporal structure of our universe (in particular, empirical evidence is now that our universe will keep on expanding, and that the presence of dark energy is responsible for this). The chapters on Zeno, however, would not have been on my own priority list if I had been in Dainton's shoes. It is true that these chapters clearly explain the intuitively strange features of uncountable sets and continuity, and that this is certainly relevant to our notions of space and time. But, updating other subjects in the book might have been more immediately relevant. In the chapter on Time Asymmetry, there is attention for entropy, causality, and how entropic and causal asymmetries may relate to the initial state of the universe, but the text does not engage with any developments after Paul Horwich's "Asymmetries in Time" (1987). However, the last decade has seen a lot of significant novel discussion about exactly these topics, in particular in connection with the so-called Past Hypothesis and the question of how to distinguish between laws and initial conditions. Further, in the chapter on Special Relativity there is a several-page explanation of Einstein's procedure for synchronizing clocks, with the conclusion that simultaneity in the special theory is relative to inertial frames. This is then used in the next chapter, Relativity and Reality, to critically discuss the notorious Rietdijk/Putnam argument according to which special relativity proves the "actuality" of the block universe. (What is present, "and therefore real" to me, is for a moving observer simultaneous with events in my future, "and therefore these future events exist already now for me".) What Dainton writes about this is clear and illuminating, but it is strange that in these chapters there is no mention at all of the well-known debate about the conventionality of relativistic simultaneity: If simultaneity is purely conventional and lacks metaphysical significance, there is 
obviously no reason to suppose that simultaneous events share a special "realityproperty", so that the Rietdijk/Putnam argument seems to become a non-starter.

Another popular subject in the recent literature that I miss in Dainton's discussion is the debate about endurance versus perdurance: Are objects totally present at any instant of time, so that they are essentially three-dimensional, or should they rather be seen as four-dimensional world-tubes with three-dimensional temporal parts? There are obvious relations here to topics that do figure prominently in the book, like Presentism versus Eternalism. Finally, the last years have seen interesting new proposals for developing theories in which space and time emerge from simpler structures. In addition to suggestions based on string theory, mentioned in the book, there are also the recent attempts by Verlinde and others to use the holographic principle, together with information-theoretic ideas, to derive both spatial notions and general relativity from non-spatial premises.

Perhaps some of these topics could be considered for the third edition of "Time and Space". But also without them the book is admirably comprehensive and sets a standard for analytical work in this subject area. It is an indispensable help for anyone wishing to become familiar with the philosophy of space and time, and I highly recommend it.

Open Access This article is distributed under the terms of the Creative Commons Attribution License which permits any use, distribution, and reproduction in any medium, provided the original author(s) and the source are credited. 